See discussions, stats, and author profiles for this publication at: https://www.researchgate.net/publication/305114954

\title{
Assistive Technologies For The Older People: Physical Activity Monitoring And Fall Detection
}

Article · July 2016

\section{CITATION}

1

6 authors, including:

7. Chi Hieu Le

University of Greenwich

72 PUBLICATIONS 373 CITATIONS

SEE PROFILE

Jin Luo

University of West London

35 PUBLICATIONS 985 CITATIONS

SEE PROFILE
READS

446

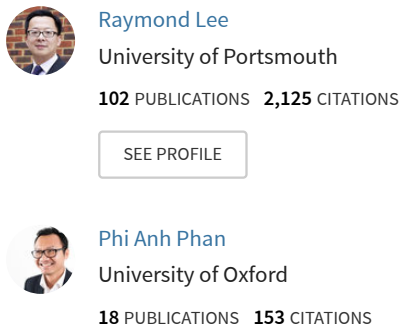

SEE PROFILE

Some of the authors of this publication are also working on these related projects:

The inspired sinewave technique View project

Innovative design and development of smart patient-specific implants and medical devices, loT, mass-customisation, telehealth, 3D medical image processing for preoperative planning, higher education (innovative learning, teaching and assessment) View project 


\title{
Assistive Technologies For The Older People: Physical Activity Monitoring And Fall Detection
}

\author{
Raymond Lee ${ }^{1}$, Jin Luo ${ }^{2}$, Chi Hieu Le ${ }^{3}$, Phi Anh Phan ${ }^{4}$, Trung Le ${ }^{5}$ and Minh Hoa Le ${ }^{6}$ \\ ${ }^{1}$ School of Applied Sciences, London South Bank University, London, United Kingdom \\ ${ }^{2}$ Department of Life Sciences, University of Roehampton, United Kingdom \\ ${ }^{3}$ Faculty of Engineering and Science, University of Greenwich, Kent, United Kingdom \\ ${ }^{4}$ The Nuffield Division of Anaesthetics, University of Oxford, Oxford, United Kingdom \\ ${ }^{5}$ International University-Vietnam National University, Ho Chi Minh, Vietnam \\ ${ }^{6}$ Faculty of Engineering and Environment, Northumbria University, Newcastle upon Tyne, United Kingdom
}

\begin{abstract}
The advancements in information and communications technologies (ICT) and micro-nano manufacturing lead to innovative developments of smart sensors and intelligent devices as well as related assistive technologies which have been directly contributing to improving the life quality, from early detection of diseases to assisting daily living activities. Physical activity monitoring and fall detection are two specific examples where assistive technologies with the use of smart sensors and intelligent devices may play a key role in enhancing the life quality, especially improving the musculoskeletal health which is an essential aspect of health and wellbeing; and it is more important for the older people. This paper presents and discusses about how sensors and wearable devices, such as accelerometers and mobile phones, may be employed to promote the musculoskeletal health. Assistive technologies and methods for physical activity monitoring and fall detection are discussed, with the focus on the fall detection using mobile phone technology, and assessments of the loading intensity of physical activity in a non-laboratory environment. The possible research directions, challenges and potential collaborations in the areas of assistive technologies and ICT solutions for the older populations are proposed and addressed.
\end{abstract}

Keywords - Assistive technology, fall detection, telehealth, older people, ageing population, physical activity monitoring.

\section{INTRODUCTION}

Physical inactivity is recognized as a major public health concern, contributing to about $9 \%$ of premature deaths and 6-10\% of prevalence of non-communicable diseases worldwide such as heart disease and type 2 diabetes [1]. Healthrelated quality of life is therefore strongly related to the physical activity (PA). Especially, the musculoskeletal health is an essential aspect of health and wellbeing throughout an individual's lifespan, but there is a decline in musculoskeletal health in both men and women as we age [2]. PA has been widely recommended as an effective intervention for the conservation of musculoskeletal health [3, 4, 22]. Energy expenditure and PA levels are the important health determinants, especially the mechanical loading intensity [6]. However, it is still unclear how much dose of mechanical loading is required to bring about improvement in the health in general and the health of bone and muscles in particular. The dose of physical activity will include factors such as the intensity and frequency of the mechanical loads [5]. PA is important in the promotion of musculoskeletal health [6,7]; however, falls often happen during physical exercise, walking and various forms of PA [8,9]. Fall is a major care and cost burden to the health and social services worldwide [10,11]. Although most falls produce no serious consequence, $5-10 \%$ of community-dwelling older adults who fall each year do sustain serious injuries such as fractures, head injuries or serious laceration [12].

Globally, $40 \%$ of older persons aged 60 years or over live independently, alone or with their spouse only; and as countries develop and their populations continue to age, living alone or with a spouse only will likely become much more common among older people in the future [19]. Therefore, Assistive technologies (AT) for monitoring and assessment of physical activities and fall detection play an important role in improving the overall well-being and life quality for older populations. Especially, AT devices help elderly people feeling safer, preventing falls, minimizing the risk of an accident and encouraging active participations in community activities. In addition, the good monitoring of physical activities helps tracking the levels of physical activities, leading towards a healthier lifestyle.

PA monitoring and fall detection are therefore two specific examples where AT may play a key role in enhancing the life quality, especially improving the musculoskeletal health and having healthier lifestyle. This paper presents and discusses about how inertial sensors, such as accelerometers, may be employed to promote musculoskeletal health which is an essential aspect of health and wellbeing; and it is more important for the older people. The rest of the paper is organized as follows. Section II presents devices for monitoring physical activities with the focus on our recent studies about assessments of the loading intensity of PA in a nonlaboratory environment. Section III presents and discusses about devices for fall detections using mobile phone technology. Finally, the discussions and conclusions are presented in Section IV, highlighting the possible research 
directions, challenges and potential collaborations potential collaborations among research institutions in UK and developing countries, including Vietnam, to innovatively develop AT with cost-effective solutions in the areas of assistive technologies and ICT solutions for the older populations.

\section{DEVICES AND METHODS FOR MONITORING PHYSICAL ACTIVITIES IN A NON-LABORATORY ENVIRONMENT}

Our research group has developed a novel method that can objectively assess the loading intensity PA in a nonlaboratory environment $[13,14]$. Using a miniature accelerometer attached to the trunk, a loading intensity can be derived from the magnitude and frequency of the signals. Our research shows that the method is able to discriminate the loading intensities of different physical activities and to look the intensities in different frequency spectra. One major attraction of this method is that it is non-intrusive and can monitor activities throughout the day, or over a long period of time.

Table 1 Correlation coefficient (Kendall's $\tau$ ) between loading dose and age, BUA, and knee extension torque (Frequency LD_B1 $=0.1-2 \mathrm{~Hz}, \mathrm{LD} \_\mathrm{B} 2=2$ 4Hz, LD_B3=4-6Hz)

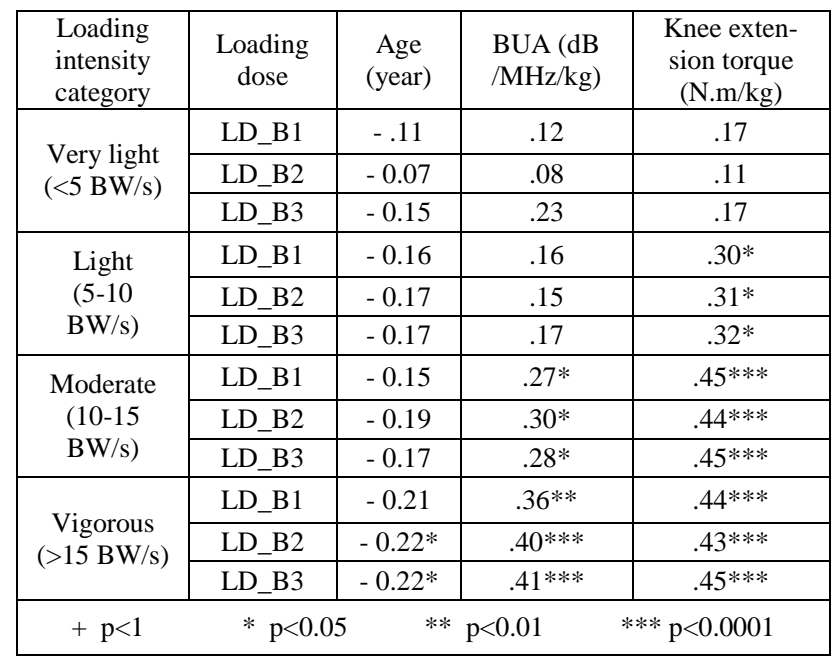

We have also investigated the association between loading dose of PA, muscle strength and bone density in a group of 34 middle-aged women [14]. They were requested to wear an accelerometer for a period of $10 \mathrm{~h}$ (from 9 am to 7 $\mathrm{pm}$ ) on a day to record the acceleration. On a separate day their knee extension torque (KET) was measured using an isokinetic dynamometer and broadband ultrasound attenuation (BUA) at the heel by an ultrasound bone scanner. The loading dose of PA was calculated at four intensity categories - very light, light, moderate, and vigorous (intensities of $<5 \mathrm{BW} / \mathrm{s}, 5-10 \mathrm{BW} / \mathrm{s}, 10-15 \mathrm{BW} / \mathrm{s}$ and $>15 \mathrm{BW} / \mathrm{s}$ ) and for three frequency bands $-0.1-2 \mathrm{~Hz}, 2-4 \mathrm{~Hz}$, and 4-6 Hz. Correlation analysis was used to examine the association between loading dose and age, KET, and BUA (Table 1). The increase in loading dose in all three frequency bands in moderate or vigorous activity was associated with higher BUA and KET (Kendall's tau $=.27-.45, \mathrm{p}<.05$ ). There is clear evidence to support that PA, especially that at high intensity level and high frequency range, may have beneficial effect on muscle strength and bone density in middleaged women.

\section{DEVICES AND METHODS FOR FALL DETECTION}

Body-mounted accelerometers have been used to detect falls in various studies $[15,16,17]$. Our research group has made use of the current mobile phone technology to detect falls as this will allow us to do additional data processing and identify the location of the fall. Since the mobile phone is not originally designed for fall detections, we therefore developed a computer program to acquire signals from the phone and detect falls, incorporating the algorithm illustrated in Fig.1.

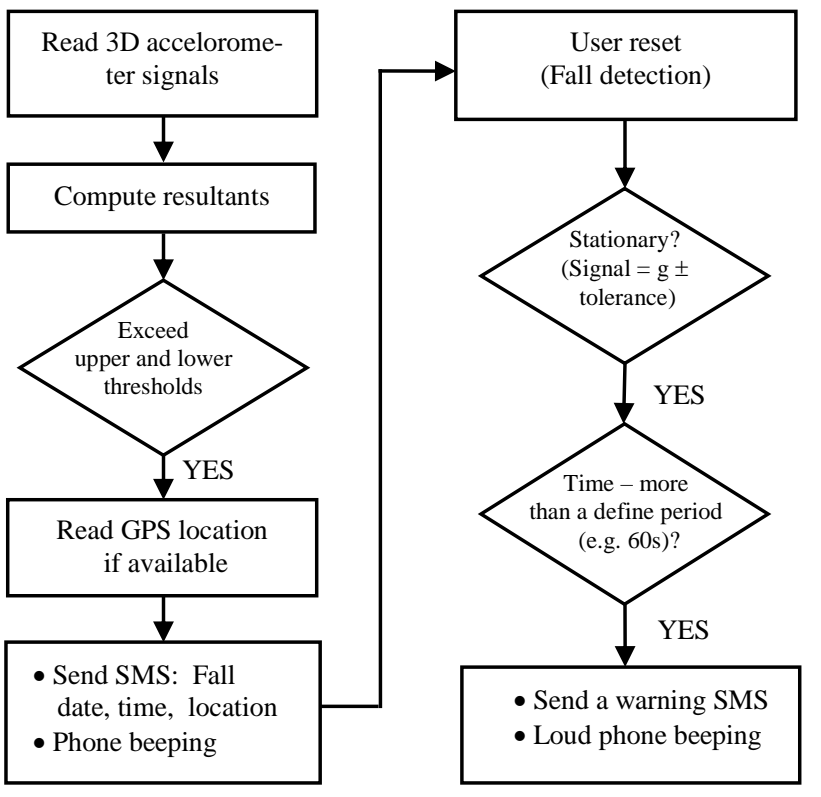

Fig.1 A block diagram showing the fall detection algorithm

In our experimental study [18], each participant was requested to perform three trials of four different types of simulated falls (forwards, backwards, lateral left and lateral right) and eight other everyday activities (sit-to-stand, standto-sit, level walking, walking up- and downstairs, answering the phone, picking up an object and getting up from supine). Acceleration was measured using two devices, a mobile 
phone and an independent accelerometer attached to the waist of the participants. Bland-Altman analysis showed a higher degree of agreement between the data recorded by the two devices. Using individual upper and lower detection thresholds, the specificity and sensitivity for mobile phone were 0.81 and 0.77 , respectively, and for external accelerometer they were 0.82 and 0.96 , respectively. We therefore suggest that fall detection using a mobile phone is a feasible and highly attractive technology for older adults, especially those living alone. It may be best achieved with an accelerometer attached to the waist, which transmits signals wirelessly to a phone.

\section{DisCUSSION AND CONCLUSION}

There is an emerging need to develop innovative and cost-effective AT products for the older populations, especially for the low-income and developing countries. Population ageing has major social and economic consequences. According to the recent report of United Nations, there is a rapid growth in ageing and older populations. The global share of older people, aged 60 years or over, increased from $9.2 \%$ in 1990 to $11.7 \%$ in 2013 and will continue to grow as a proportion of the world population, reaching $21.1 \%$ by 2050 ; and it is from 841 million people in 2013 to more than 2 billion in 2050 [19]. The years lived with disability due to falls in adults aged 50-69 in developing countries $(66 \%)$ was nearly double the rate in developed countries (34\%) [20].

In developing countries, poverty is high among older persons, sometimes higher than that of the population as a whole, especially in countries with limited coverage of social security systems; and diseases and disability increase as populations age [19]. Cost-effective solutions and AT for monitoring the health status, early detection of disease, preventing the risks to disabilities, and assisting the daily living activities are very important and necessary to improve the life quality of ageing and older populations.

With recent advancements in ICT and micro-nano manufacturing, smart sensors and intelligent devices are innovatively developed that play a key role in enhancing musculoskeletal health which is an essential aspect of health and wellbeing; and it is more important for the older people. In combination with Internet of things (IoT), labs-on-chips and telehealth technologies, it is possible and potential to develop a new AT generation of wearable intelligent products that are able to provide powerful functions for PA monitoring, fall detection and prevention, health risk predictions, early disease detection and assessment, to directly assist the daily living activities and improve the life quality of ageing and older populations, especially to help them feeling safer, preventing falls, minimizing the risk of an accident and encouraging active participations in community activities.
In this paper, two specific examples where AT with the use of smart sensors and intelligent devices are presented and discussed, aimed to enhance the life quality of older populations, via physical activity monitoring and fall detection. This broad area of Biomedical Engineering brings together a number of disciplines to manage various clinical conditions, including osteoporosis, osteoarthritis, stroke, spinal cord injuries, post-operative care and trauma. The demographic shift to an increasingly older population in various countries, including the low-income and developing countries, will mean that this research area will continue to be of strategic priority.

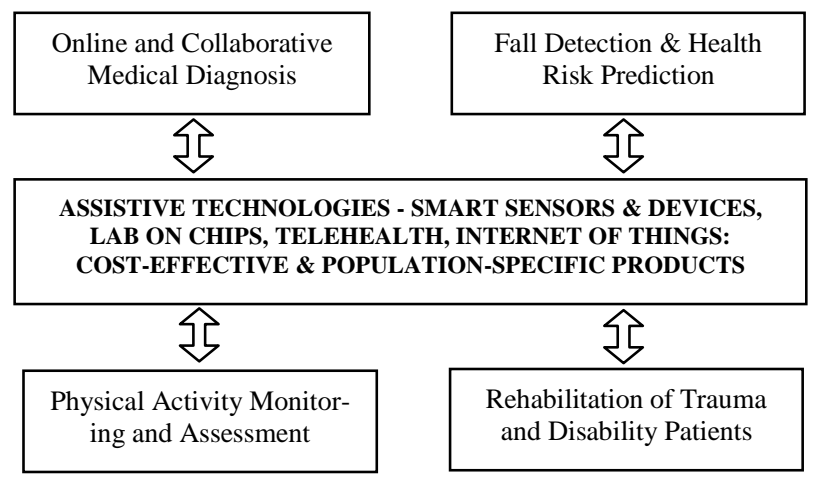

Fig. 2 A proposed research areas for the potential collaborations among research institutions in UK and developing countries.

In the UK, the research base has been fuelling the growth of the medical technology sector with a combined annual turnover of $£ 15$ billion; and the funding from Engineering and Physical Science Research Council (EPSRC) for healthcare is up to $75.9 \%$ [21]. In the areas of AT for older populations, the UK research community has the potential to transform research in the following areas: next generation prosthetics and implants, population-specific diagnosis and treatments, assistive devices and wearable products for PA monitoring and fall detection, health risk predictions and prevention, improved neuromusculoskeletal models addressing patient variance, IoT, labs-on-chips and telehealth technologies. These lead to potential research and technology collaborations as well as commercialisation among research institutions in UK and developing countries, including Vietnam, to innovatively develop AT with cost-effective solutions, based on the use of the local resources and technology infrastructure, as well as the effective collaboration between the engineers, neuroscientists, clinicians, industrial partners and users.

Figure 2 presents the proposed research areas for the potential collaborations among research institutions in UK and developing countries. Specifically, within the scope of research in this paper, the potential collaborations among research institutions in UK and developing countries include the following proposed topics: 


\section{(a) Detection of falls for the real fall situations}

It is necessary to develop methods and cost-effective AT to detect falls for the real fall situations, incorporating subtle fall events, and falls during more vigorous activities such as jogging, especially development of the more sophisticated and robust detection algorithm to deal with the more complex activities. More advanced and smart sensors need to be developed with better sensitivity and functions.

\section{(b) Prediction and detection of falls with the use wearable products or mobile phones}

With the rapid advancements of ICT, IoT and micro-nano manufacturing, it is possible to develop cost-effective wearable products for fall detection and prevention, via the use of integrated smart sensors that attached to the currently available wearable products or mobile phones. The use of a small external accelerometer wirelessly connected to a mobile phone or wearable product via Bluetooth may be an option to increate the sensitivity of fall detections and reduce the manufacturing cost.

\section{(c) Innovative development of AT devices and technological solutions to monitor and assess PA as well as early de- tection of diseases and health-risk presentation}

Since health-related quality of life is indirectly or directly related to PA. In addition, PA is an effective intervention for the conservation of musculoskeletal health. The required amount of physical activity depends on the age. It is important and necessary to develop AT devices and technological solutions that can accurately measure the parameters that are determinants for bone and muscle adaptation, such as intensity, frequency, and dose of mechanical loading. This also leads to the development of effective devices and methods to monitor the administration of exercise programme and to provide useful feedback and information about the loading intensity and frequency of PA required to bring about positive effects on the health. Records of PA and medical tests may be useful for early detection of diseases and health-risk presentation.

\section{ACKNOWLEDGMENT}

British Council-Newton Fund is acknowledged for their support.

\section{REFERENCES}

1. Lee I.M et al. (2012) Physical Activity Series Working Group. Effect of physical inactivity on major non-communicable diseases worldwide: an analysis of burden of disease and life expectancy. Lancet; 380, 219-229
2. R.T. Kell, G. Bell and A. Quinney (2001) Musculoskeletal fitness, health outcomes and quality of life Sports Med; 31: 863-873

3. M.C. Walsh and G.R. Hunter (2006) Livingstone Sarcopenia in premenopausal and postmenopausal women with osteopenia, osteoporosis and normal bone mineral density Osteoporos Int; 17: 61-67

4. T. Akune et al. (2014) Exercise habits during middle age are associated with lower prevalence of sarcopenia: the ROAD study Osteoporos Int; 25: 1081-1088

5. C.H. Turner (1998) Three rules for bone adaptation to mechanical stimuli Bone; 23: 399-407

6. Department of Health. The health benefits of physical activity: www.dh.gov.uk [Accessed April 2016].

7. Oakley A et al. (1996) Preventing falls and subsequent injury in older people. Qual Health Care; 5:243-249

8. Iinattiniemi S, Jokelainen J, Luukinen H. (2008) Exercise and risk of injurious fall in home-dwelling elderly. Int $\mathrm{J}$ Circumpolar Health; 67:235-244

9. Talbot LA, Musiol RJ, Witham EK, et al. (2005) Falls in young, middle-aged and older community dwelling adults: perceived cause, environmental factors and injury. BMC Public Health; 5:86.

10. Annekenny R, O'Shea D. (2002) Falls and syncope in elderly patients. Clin Geriatric Med; 18:xiii-xiv

11. Scuffam P, Chaplin S, Legood R. (2003) Incidence and costs of unintentional falls in older people in the United Kingdom. J Epidemiol Community Health; 57:740-744

12. Tinetti ME, Doucette J, Claus E, et al. (1995) Risk factors for serious injury during falls by older persons in the community. J Am Geriatrics Soc; $43: 1214-1221$

13. S. Kelley, G. Hopkinson, S. Strike, J. Luo, R. Lee. An accelerometry based approach to assess loading intensity of physical activity on bone Res Q Exerc Sport; 85: 245-250

14. J Chahai, R Lee and J Luo (2014) Loading dose of physical activity is related to muscle strength and bone density in middle-aged women. Bone; 67:41-45.

15. M Kangas, I Vikman, J Wiklander, et al. (2009) Sensitivity and specificity of fall detection in people aged 40 years and over. Gait Posture, 2009;29:571-574

16. A Kumar, F Rahman and T Lee. (2009) Fall detection unit for elderly. 13th International Conference on Biomedical Engineering, 2009, pp. 984-986

17. J Boyle, MKarunanithi. (2008) Simulated fall detection via accelerometers. Conf Proc IEEE Eng Med Biol Soc., 2008: 1:81274-1277

18. R Lee, A Carlisle (2011) Detection of falls using accelerometers and mobile phone technology. Age and ageing; 40(6):690-696.

19. United Nations, Department of Economic and Social Affairs, Population Division (2013). World Population Ageing 2013. ST/ESA/SER.A/348

20. Keshini M.M. (2015) Assistive technologies for ageing populations in six low-income and middle-income countries: a systematic review. BMJ Innovations; 0:1-14

21. EPSRC (2013) Assistive technology, rehabilitation and musculoskeletal biomechanics: www.epsrc.ac.uk [Access: April 2016]

22. K.T. Borer (2005) Physical activity in the prevention and amelioration of osteoporosis in women: interaction of mechanical, hormonal and dietary factors Sports Med; 35: 779-830

Author: Raymond Lee

Institute: London South Bank University

Street: Borough

City: London

Country: United Kingdom

Email: r.lee@1sbu.ac.uk 